their families and carers. We are keen to share our results and experience with others and to improve further our service.

\section{P155 MAKING SENSE OF SHADES OF GREY!: IMPACT OF HOSPICE ULTRASOUND USE ON PATIENT CARE}

Tim Jones, Karen Groves. Queenscourt Hospice, Southport, UK

10.1136/bmjspcare-2013-000591.177

Background Patients with advanced disease require transportation to hospital for ultrasound identification of abnormal fluid and location marking. This is not only exhausting, but introduces service delays, increases work for an already overburdened hospital radiology service and reproducing the exact position of original scan is difficult.

Aim Does the introduction of portable ultrasound improve patient, carer $\&$ health professional experience and co-ordination of care?

Method Two hospice doctors underwent the necessary ultrasound scanning training for non radiologists. Following a selection process, an appropriate portable ultrasound machine was purchased, and support agreed with one of the local radiologists. Results A retrospective review of thirty months ultrasound use was undertaken. One hundred and four patients have had one hundred and sixty eight separate scanning episodes resulting in eighty five procedures. Uses have included confirmation of presence \& location of abdominal ascites prior to paracentesis, confirmation of location of pleural fluid prior to aspiration, confirmation of full bladder prior to catheterisation (urethral or suprapubic), monitoring the growth of large intra-abdominal malignant cyst, confirmation of hydronephrosis, biliary stent placement $\&$ locating nerve in real time neural blockade. The poster includes detailed analysis of use.

Conclusion On each occasion a definitive clinical question was answered. Patients report greater satisfaction from a speedier service, families feel that patients are less fatigued by the interventions and staff feel able to give a better, more responsive, safer service, adhering to current best practice guidance. Being able to ultrasound patients in the home or in outpatients has prevented unnecessary admissions where the cause of abdominal distension had previously been in doubt.

\section{P156 LUNG CLINIC TO MEET THE NEEDS OF PATIENTS WITH NON MALIGNANT LUNG DISEASE AT GARDEN HOUSE HOSPICE}

Diane Wallis. Garden House Hospice, Letchworth, UK

10.1136/bmjspcare-2013-000591.178

Introduction In 2011 the Department of Health Outcomes strategy for COPD suggested that the needs and preferences of patients for care at the end of life were not being met.

The model of Day Hospice did not meet the needs of this group of patients; therefore a proposal was put forward with the support of the local chest physicians to provide a service accessible to COPD patients and their carers.

A pilot clinic was set up for twelve months. Staffed by a nursing Sister, a physiotherapist and trained volunteers.

Aims The aim was to develop an accessible multidisciplinary service that met the individual needs of the patients, family and carers. This included transition to palliative care services, psychosocial support, symptom management and advanced care planning.

Method During the pilot and the following year the numbers of patients attending was monitored. Also the source of referrals was examined. Some anecdotal feedback was gained from the referrers. The service was adjusted overtime to maximise accessibility for the patients.

A qualitative questionnaire was produced in December 2012 to find out the perceived benefits for patients attending the clinic and if there was anything else that could be provided. Nine questionnaires were completed.

Results The results of the questionnaire suggested that the patients were fearful of attending the hospice beforehand, however, once attendance had commenced their psychosocial and symptom control needs were being met. The results of the questionnaire will be made available.

Conclusion The clinic is meeting the patients' needs, therefore it will continue in its current format. There is a need to discuss and consider strategies to educate the referrers on Hospice philosophy and the aims of the clinic so they can help change the fearful expectations of the patients on referral.

\section{P157 A PALLIATIVE CARE INTERVENTION FOR PATIENTS WITH ADVANCED NON-MALIGNANT RESPIRATORY ILLNESS}

${ }^{1}$ Marjory Mackay, ${ }^{1}$ Janice Christie, ${ }^{1}$ Alison Moodie, ${ }^{2}$ Deborah Lynch. ${ }^{1}$ Strathcarron Hospice, Denny, Scotland, ${ }^{2}$ NHS Forth Valley

\subsection{6/bmjspcare-2013-000591.179}

Introduction Patients with advanced respiratory disease do not routinely access palliative care services and given that many are unable to attend hospital appointments, they are even less likely to receive quality palliative care support in their last year of life. This collaborative project between an NHS Respiratory Team and a Hospice aimed to address this inequity.

Aims The specific project aims were to: improve symptom control; raise patient and carer awareness /understanding of illness and prognosis; explore patients' wishes about future treatment and care and establish an advance care plan (ACP); communicate patients' wishes across health care settings; reduce inappropriate hospital admissions and inappropriate treatments.

Methods Using a convenience sample over a 12 month period, all patients who met inclusion criteria (adapted from Gold Standard Framework prognostic indicator guidance) were referred by the respiratory team to specialist palliative care $(\mathrm{n}=$ 49). The planned intervention comprised three visits to the patient's home by a palliative care nurse specialist.

Data collection aimed to evaluate the outcome of visits; the follow-through of ACP wishes during hospital admissions; patient experience; GP feedback; impact of project on specialist respiratory nurses and patterns of hospital admissions.

Quantitative data was analysed using descriptive statistics and Qualitative data was collated into themes.

A favourable ethical opinion was granted for the project.

Results Results to date suggest that 1) selection criteria were effective, 2) the intervention had a positive effect on patients' wellbeing and engagement with the ACP process, 3) purposeful communication and treatment across healthcare settings were enhanced. Some challenges are also emerging, all of which will help shape future service provision. It is anticipated that this collaborative model of service is transferrable to other non-malignant specialties and could be adapted by other hospices / 